\title{
EVALUACIÓN DE LA RECUPERACIÓN DE ESTRUCTURAS DE CONCRETO ARMADO POR REMOCIÓN SÓLO DE LA CAPA AFECTADA DEL RECUBRIMENTO DE LA ARMADURA.
}

\section{Evaluation of repair in reinforced concrete when only take off the coating layer of de Steel bars.}

\section{Doctorando:}

\section{José Emidio Alexandrino Bezerra}

Universidade de Fortaleza, Facultad Regional Córdoba, Universidad Tecnológica Nacional - Argentina joseemidiobezerra@outlook.com

\section{Director/es: \\ Antônio Eduardo Bezerra Cabral}

Co-director/es:

\section{Angel Oshiro}

\section{Resumen}

En esta investigación se utilizaron especímenes prismáticos moldeados con concreto, mortero estructural, mortero polimérico y graute para representar los reparaciones cuando se remueve todo concreto envuelta de la barra afectada. Las muestras también se hicieron con reparaciones solo en la capa de recubrimiento de barras de acero para representar reparaciones parciales. Cada CPP con dos varillas de acero CA $60 \emptyset 5.0 \mathrm{~mm}$ con pintura protectora a base de zinc, pintura protectora a base de nitrito y sin protección. Las propiedades físicas de los materiales se midieron en probetas cilíndricas. Los CPP se sometieron a ciclos de secado durante cinco días en un horno a 50 ํㅡ, seguidos de dos días de humectación por inmersión parcial en solución de $\mathrm{NaCl}$ al 3,5\%. Después de cada ciclo, los potenciales de corrosión se midieron con equipos con voltímetro de alta impedancia y semicélula de cobre / sulfato de cobre. Se obtuvieron datos que permitieron comparar la eficiencia dela la protección de zinc y de nitritos recubiertos con concreto, mortero estructural, mortero polimérico y graute. El análisis preliminar, encontró que la protección con nitritos y graute cementosa obtuvo los mejores resultados y que remover todo el concreto alrededor de las barras de acero la protección es mejor, sin embargo, las reparaciones parciales con inhibidores de corrosión y recubrimiento con mortero polimérico o graute, todavía, proporciona una buena protección, ofreciendo menos riesgo a la estructura. Palabras clave: métodos electroquímicos, potencial de corrosión, mortero, concreto, cloruros, nitritos.

\footnotetext{
Abstract

In this investigation, there were used prismatic specimens (CPP) molded with concrete, structural mortar, polymeric mortar and grout, In order to represent the repairs when all concrete is removed around the affected bar. In addition, samples made with repairs only to the steel bar cover layer to represent partial repairs. Each CPP with two CA $60 \varnothing 5.0 \mathrm{~mm}$ steel rods inserted. prepared in three conditions: zinc-based protective paint, nitrite-based protective paint and no protection. The physical properties of the materials, such as compressive strength, water absorption, and electrical resistivity, measured on cylindrical specimens. The CPPs subjected to drying cycles for five
} 
days in an oven at $50^{\circ} \mathrm{C}$, followed by two days of wetting by partial immersion in 3.5\% NaCl solution. After each cycle, the corrosion potentials measured with equipment with a high impedance voltmeter and a copper / copper sulfate half-cell. Data obtained that allowed to compare the efficiency of the protection as zinc and with nitrites coated with concrete, structural mortar, polymeric mortar and free against the attack of chloride. Thus, it seeks to discover which material has a better performance and which the degree of disadvantage between total and partial repairs. The preliminary analysis found that the protection with nitrites and cementitious grout obtained the best results, that when removing all the concrete around the steel bars the protection is better, however, partial repairs with corrosion inhibitors and coating with polymeric mortar or grout provides good protection, offering less risk to the structure. Keywords: electrochemical methods, corrosion potential, mortar, concrete, chlorides, nitrites.

\section{Introducción}

La corrosion del refuerzo de hormigón se debe principalmente a la carbonatación del hormigón y la interacción con los cloruros. A este respecto, Helene (2014) afirma que la pérdida de la protección natural de la armadura por el revestimiento de hormigón puede ocurrir a través de varios mecanismos, siendo predominante la despasivación por carbonatación y por la acción de iones de cloro. Battagin (2010) citado por Borges (2015) afirma que los agentes agresivos que atacan la pasta de cemento en el hormigón son las sales y sulfatos de magnesio, mientras que los cloruros son los responsables de la corrosión de la armadura. Los cloruros, por sus muy reducidas dimensiones tienen gran movilidad dentro del hormigón, pueden llegar fácilmente a las armaduras.

GIUDICE (2016) afirma que la corrosión electroquímica es en realidad una red de celdas galvánicas en cortocircuito colocadas sobre la superficie metálica y, como explica Rüsch (1975) y citado por Helene (1976), este mecanismo requiere la existencia de un conductor, electrolito, diferencia de potencial eléctrico y oxígeno que se producirán y mantendrán a la continuación.

Este proceso de corrosión del acero en el hormigón tiene una fase inicial, en la que agentes agresivos modifican las condiciones del hormigón alrededor de la barra, haciéndola menos pasiva y permitiendo así la formación de una celda de corrosión, responsable de la propagación de la corrosión. (Pazini y Meira, 2013) Ellos explican que los cloruros son los más destructivos debido a su abundancia en las áreas costeras donde se ubican muchas estructuras de construcción y porque no se consumen en las reacciones de oxi-reducción.

Las reparaciones estructurales son muy importantes y debe hacerse con cuidado. Diversos autores recomiendan la remoción de todo el hormigón afectado por la corrosión de la armadura como condición sine qua non para realizar la deseada reparación. Otros autores mencionan que dependiendo de la extensión del corte y la capacidad resistente residual puede ser necesario apuntalar el elemento estructural, a pesar que en muchos casos es dificultoso por los trastornos que puede causar a los usuarios de los inmuebles residenciales y comerciales.

Aunque autores y normas indican que se debe remover todo el hormigón alrededor del perímetro de la barra oxidada, es habitual por parte de profesionales y empresas, remover sólo la capa de recubrimiento sin desplazar el hormigón por detrás de la barra cuando la corrosión no es muy intensa. Así, la desoxidación es hecha solamente en la parte expuesta de la barra, dejando inmersa en el hormigón original la parte posterior de la misma.

Seguro que no se puede negar la importancia de la remoción de agentes agresivos en la región de las fallas, pero algunas veces puede ser peligroso. Entonces, acá si propone evaluar cuál es la pérdida de cualidad cuando solo se quita la capa de cubrimiento de las barras de acero y que solución presenta la mejor relación costo/beneficio para prolongar la vida útil de las estructuras con reparaciones hechas sobre barras de acero no totalmente desoxidadas.

Además, no toda la corrosión del refuerzo de concreto se debe a la presencia de cloruros, muchos se deben a la carbonatación, que no ataca el acero, solo elimina la protección alcalina del concreto para él. Por el expuesto, resulta razonable y, en principio relevante, estudiar y evaluar la eficiencia y la eficacia de las reparaciones con la remoción parcial del hormigón afectado.

Por ello, es relevante estudiar la efectividad de los materiales utilizados en las reparaciones estructurales y, para ello, se pueden utilizar ensayos no destructivos, tales como la medición de la resistividad del material, potenciales de corrosión y difusión de cloruros. La resistividad se puede utilizar para estimar la probabilidad de corrosión y de acuerdo con CEB 192/88 para cemento Portland a $20^{\circ} \mathrm{C}$.

La técnica de velocidad de pulso ultrasónico, según Ribeiro y Cunha, (2018) permite obtener información del módulo de elasticidad y de la resistencia a la compresión, pudiendo identificar huecos en el interior del material. Al respecto, Posetiere y Gioia (2017) aclaran que el ultrasonido es una onda que supera los $20 \mathrm{KHz}$ y supondría una perturbación en el entorno en el que se propaga, provocando que las moléculas y átomos vibren de forma elástica. Por tanto, la velocidad del pulso está relacionada con la calidad del hormigón y según la norma inglesa BS EM 1254-4/04). 
El método de medición del potencial de corrosión mide la carga negativa provocada por el movimiento de los electrones liberados por los iones ferrosos que se mueven a través del electrolito formado en el hormigón, proporcionando así información sobre el estado de corrosión de la armadura, según ZERBINO y CARRASCO (2012).

\section{Desarrollo}

El programa experimental fue planejado para obtener medidas de los potenciales de corrosión en probetas prismáticas de mortero estructural, mortero polimérico, graute y hormigón con Fck $=24 \mathrm{MPa}(\mathrm{NBR}$ 9779/12) que servirá como base de referencia. Fueron sometidos a mediciones semanales para medir la evolución de la corrosión potencial, en 12 ciclos de secado ( 5 días en horno a $50^{\circ} \mathrm{C}$ ) y humedecimiento en solución de NaCL al 3.5\% ( 2 días).

\section{Caracterización de los materiales constituyentes}

Los datos relativos a los materiales utilizados para la preparación del hormigón utilizado en las probetas son los siguientes:

Cemento Portland CP II F 32, compuesto con adición de masilla de caliza entre 6\% y 10\%. Blaine superior a 2600 cm2 / g, resistencia a la compresión> 32 MPa a los 28 días y cumple con NBR 16697/18.

Arena gruesa del río Chorozinho, en la localidad de Criancó/Ceará con las características expuestas en la Tabla 1

\begin{tabular}{|c|c|c|}
\hline Ensayo realizado & Resultado & Norma de ensaio \\
\hline Módulo de finura & 2,77 & NBR 7217/87 \\
\hline D máx. característica & $2,36 \mathrm{~mm}$ & NBR $7217 / 87$ \\
\hline Massa específica & 2,62 & NBR $9776 / 87$ \\
\hline Massa unitária & $1,48 \mathrm{~g} / \mathrm{cm} 3$ & NBR $7251 / 82$ \\
\hline Teor de materiais pulverulentos & $2,0 \%$ & NBR 7219/87 \\
\hline
\end{tabular}

- Hormigón en la mezcla 1: 2: 3 en peso con factor de agua/cemento de 0,6 y consumo de cemento de $344 \mathrm{~kg} / \mathrm{m} 3$, por ser el más utilizado en estructuras con treinta años de construcción.

- Mortero estructural industrializado, compuesto por cemento Portland y aditivos, para reparaciones estructurales de 3 a $7 \mathrm{~cm}$. (VEDACIT, 48‥ Ed.)

- Mortero polimérico industrializado, cementoso con agente adhesivo acrílico tiendo consistencia tixotrópica.

- Graute o lechada industrializada, compuesta de cemento, agregados minerales y aditivo fluidificante, libre de cloruros y resistente a sulfatos.

- Acero CA 60 se fabrica a partir de alambrón (producto laminado en caliente). Es un acero bajo en carbono que tiene una microestructura compuesta de perlita y ferrita endurecida por el proceso de deformación en frío. (ABNT, NBR 7480: 2007)

Con estos materiales se moldearon cuerpos cilíndricos (sin acero) de hormigón, graute, mortero polimérico y mortero estructural según NBR 7215/96 para los ensayos de caracterización de absorción por inmersión (NBR 9778/05), absorción de capilaridad (NBR 9779/12), difusión de cloruros (ASTM). 1202/04 y ASTM C 1556/04), ensayos mecánicos como resistencia a la compresión (NBR 9779/12). y módulo de elasticidad para mortero estructural, mortero polimérico y lechada de cemento obteniendo los siguientes resultados: (Tabla 2).

\begin{tabular}{l|l|l|l|l}
\hline \multirow{2}{*}{ Ensayos / Material } & Concreto & Mortero & Mortero & Graute \\
\cline { 2 - 4 } & $1: 2: 3$ & Estructural & Polimérico & Cemento \\
\hline Absorção de água por capilaridade (g/cm2). S=19,64 & 0,47 & 0,38 & 0,26 & 0,31 \\
\hline Absorção de água por imersão. 72 hs. Ref (\%) & 7,96 & 12,64 & 6,68 & 8,13 \\
\hline Índice de vazios. 72 hs. Ref. (\%) & 20,8 & 23,89 & 6,28 & 17,54 \\
\hline Massa específica da amostra seca. 72 hs. Ref (\%) & 2,16 & 1,89 & 2,53 & 2,16 \\
\hline Massa específica da amostra saturada. 72 hs. Ref (\%) & 2,34 & 2,14 & 2,69 & 2,33 \\
\hline
\end{tabular}

Tabla 2: Carcteristicas de los materiales. Fuente: Autor (2019)

Para la medición de la velocidad del pulso, se utilizó un dispositivo emisor / receptor de pulso ultrasónico, siguiendo el principio del dispositivo Werner.

Las resistencias a la compresión características se obtuvieron mediante pruebas de rotura en la prensa del Laboratorio de Materiales de Construcción de la Universidad de Fortaleza. 
Los potenciales de corrosión se están midiendo con equipos que tiene voltímetro de alta impedancia y semicélula de cobre/sulfato de cobre.

\section{Ensayo de corrosión por inducción acelerada de cloruro}

Se han empleado probetas prismáticas de hormigón armado con dos barras de acero para evaluar la eficiencia de las reparaciones con mortero estructural, mortero polimérico e graute, empleando sistemas de pinturas ricas en zinc y nitritos. Figura 1.

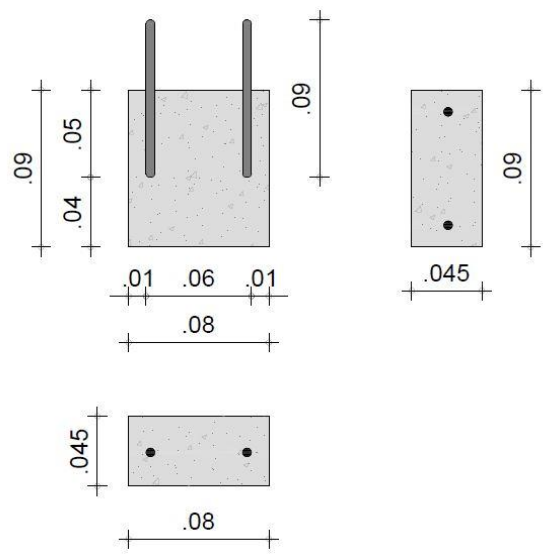

Figura 1: Probeta de concreto, morteros e lechada com dos varilla de acero. Fuente: El autor.

Las barras de acero se limpiaron con una solución de ácido clorhídrico 1: 1 con hexametilanetetramina al 3,5\%, según ASTM G - 1/03. Posteriormente, un tercio de las barras se pintaron con un producto a base de zinc y otro tercio se pintaron con un producto a base de nitritos a una longitud de $5 \mathrm{~cm}$, dejando $4 \mathrm{~cm}$ sin pintar. El resto quedó sin pintar.

Las probetas fueran sometidas a 12 ensayos acelerados de corrosión inducidos por el ion cloruro a través de ciclos de humectación y secado, hasta el 16 de diciembre de 2019. También, se está haciendo ensayos de carbonatación, pero es uno proceso más largo.

Los ensayos se realizaron en los Laboratorios de Materiales de Construcción en la Universidade de Fortaleza (Unifor) y Universidade Federal del Ceará en Brasil.

De esta forma, la corrosión es inducida por la acción de los cloruros de manera acelerada como lo verifican varios investigadores, CABRAL (2000), CASCUDO, (1991); MONTEIRO, (1996); VASCONCELOS, (1997).

\section{Resultados del ensayo de ataque por cloruros}

Para avaluar la cualidad de los reparaciones totales e parciales con protección de pinturas con zinc e nitritos e cubrimientos con morteros estructural, mortero polimérico e graute han hecho (12) ciclos de secada y humedad.

Los resultados obtenidos permitieron hacer gráficos y determinar las líneas de tendencia de los potenciales de corrosión y su cruce con la línea de -350 mV, como ejemplo, se muestra el lo gráfico 1.

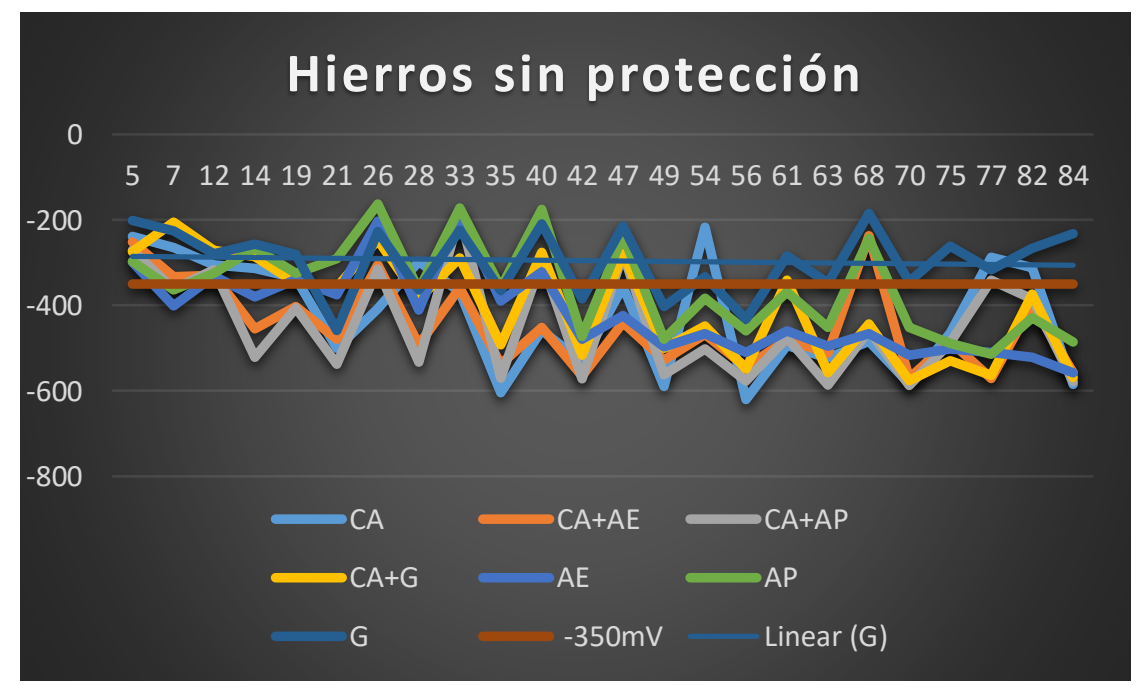

Gráfico 1: Potenciales de corrosión. Acero sin protección 
Así se obtuvo los datos, y se tiene la Tabla 3:

\begin{tabular}{|c|c|c|c|c|c|}
\hline \multirow{2}{*}{$\begin{array}{c}\text { Tipo del } \\
\text { reparo }\end{array}$} & \multirow[t]{2}{*}{ CPP/Reparo } & \multicolumn{3}{|c|}{ Días para corroer } & \multirow{2}{*}{$\begin{array}{c}\text { Mejor } \\
\text { desempeño }\end{array}$} \\
\hline & & $S P$ & $\mathrm{Ni}$ & $\mathrm{Zn}$ & \\
\hline \multirow{4}{*}{ total } & $\mathrm{CA}$ & 12 & 26 & 28 & $\mathrm{Zn}$ \\
\hline & $\mathrm{AE}$ & 21 & 61 & 35 & $\mathrm{Ni}$ \\
\hline & $A P$ & 42 & 85 & 61 & $\mathrm{Ni}$ \\
\hline & G & 85 & 63 & 47 & SP \\
\hline \multirow{3}{*}{ parcial } & $C A+A E$ & 5 & 14 & 25 & $\mathrm{Zn}$ \\
\hline & $C A+A P$ & 5 & 30 & 26 & $\mathrm{Ni}$ \\
\hline & $\mathrm{CA}+\mathrm{G}$ & 30 & 85 & 49 & $\mathrm{Ni}$ \\
\hline
\end{tabular}

En el Gráfico 2, abajo, si puede percibir que con el acero protegido con nitritos ou zinc, los reparaciones parciales resisten más al ataque de cloruros que las reparaciones totales con concreto y mortero estrutural.

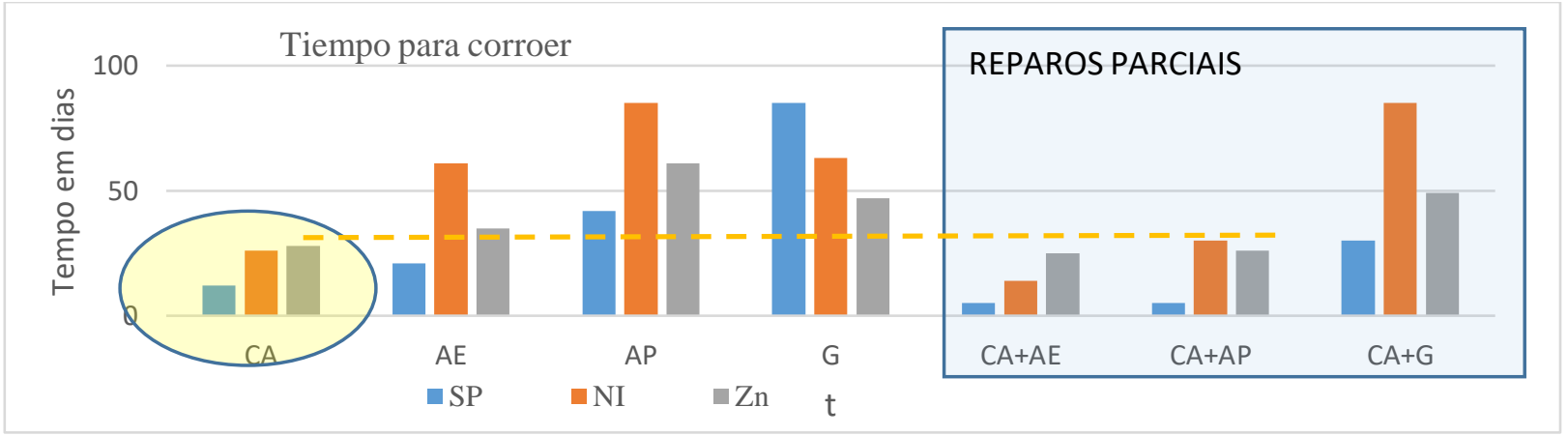

Gráfico 2 Tiempo para corroer en reparos parciales y totales.

Tambien fueron verificadas las pérdidas de masa en las varillas de acero después de 12 ciclos de secado e humedecimiento en solución de NaCL a 3,5 \%, tuvieron el seguiente comportamiento

- Hierros sin protección $1,43 \%$

- Hierros protejedos con nitritos $1,19 \%$

- Hierros protejedos con zinc $1,55 \%$

Nuevamiente, el inhibidor de protección con nitritos tuvo el mejor desempeño, considerando las varillas insertas en concreto, mortero polimérico e graute de cemento y también en las probetas de concreto parcialmente reparadas con mortero y graute, pero la diferencia entre varillas sin protección o protegidas con zinc es pequeña,

\section{Conclusiones}

Se ha podido observar que utilizando inhibidor de corrosión se obtienen resultados por encima del estado de los hierros en el hormigón original de cuando fueran ejecutados. Por lo que conviene siempre realizar la reparación, sea total o parcial, pero haya que si considerar los riesgos de colapso de la estructura cuando se remove mucho concreto en la búsqueda de la reparación perfecta, pués retirar todo el hormigón alrededor de las barras puede provocar una pérdida del 30 o 40\% de la resistencia de la columna, exponiendola a un riesgo muy grande.

Tiene que considera que una patología en una losa no provoca la demolición de un edificio y excepcionalmente y, tan poco la rotura de una viga puede provocar ésto. Pero, una ruptura en uno pilar puede causar fácilmente la destrucción de uno edificio. Afirmar que siempre tiene que remover el concreto alrededor de la barra afectada es una afirmación genérica y peligrosa, especialmente para los ingenieros con poca experiencia en estos servicios.

Tambien se tiene que se considerar que si existe una fisura con corrosión en el pilar de una edificación construdo hace 40 años y que la armadura de la columna está formada por barras de acero de 12,5 $\mathrm{mm}$ y, las barras presentan un desgaste de $1 \mathrm{~mm}$, entonces para romper la varilla serán aproximadamente 375 años! Como la mayoría de las grietas ocurren en la parte inferior de los pilares, en la región de superposición de las varillas, donde el número de barras es el doble, por lo tanto, este tiempo será aún mayor.

Suponiendo que si una reparación parcial tiene una duración de 20 años y, a continuación, se realiza otra reparación que puede durar otros 20 años, habrá un aumento de 40 años de vida útil para la estructura de un edificio. 
Las investigaciones han demostrado que las reparaciones parciales, aunque que no son tan perfectas para la patología, prolongan la vida útil de una estructura como un sistema completo.

Desafortunadamente, ya ha habido casos en los que las estructuras colapsaron parcial o totalmente debido a una recuperación localizada demasiado entusiasta.

Es verdad que el conocimiento técnico y científico sobre el fenómeno de la corrosión y recuperación estructural de acuerdo con los mejores procedimientos y estandarización es sumamente importante, sin embargo, en la práctica, estos deben sumarse a la visión integral y sistemática y al sentido común en la toma de decisiones. en la conducción y dirección de las obras.

Finalmente, es necesario pensar que la función del Ingeniero siempre será garantizar la preservación de la estructura en cualquier estado que se encuentre y conducir los servicios para tener la máxima qualidad y durabilidad no menor prazo e menor costo. Por tanto, tiene que analizar el estado dela estructura y pensar en la patología como un detalle, que debe tratarse de la mejor manera possible pero que jamais provoque el colapso de la estrutura.

\section{Referencias}

NBR 6118. (2014). Projeto de estruturas de concreto armado - Procedimento. Rio de Janeiro: Associação Brasileira de Normas Técnicas - ABNT.

NBR 9204. (2013). Concreto endurecido - Determinação da resistividade elétrica volumétrica - Método de ensaio. Rio de Janeiro: ABNT.

NBR 8522. (1984). Módulo de elasticidade. Rio de Janeiro: ABNT.

NBR 5738. (2015). Concreto - Procedimento para moldagem e cura de corpos de prova. Rio de Janeiro: ABNT.

NBR 5739. (2007). Concreto - Ensaio de compressão de corpos de prova cilíndricos. Rio de Janeiro: ABNT.

NBR 7211. (2005). Agregados para concreto - Especificação. Rio de Janeiro: ABNT.

NBR 8802. (2013). Concreto endurecido - Determinação da velocidade de propagação de onda ultrassônica. Rio de Janeiro: ABNT.

NBR 95739. (1993). Migração por cloretos, ensaios mecânicos como a resistência à compressão. Rio de Janeiro: ABNT. NBR 9778. (2005). Argamassa e concreto endurecidos - Determinação da absorção de água, índice de vazios e massa específica. Rio de Janeiro: ABNT.

NBR 7480. (2018). Aço destinado a armaduras para estruturas de concreto armado - Especificação. Rio de Janeiro: ABNT.

ASTM - C 876: 1991. Standard Test Method for Corrosion Potentials of Uncoated Reinforcing Steel in Concrete corrosion potential.

ASTM - G1: 2003. Preparing, cleaning and evaluating corrosion test especimens.

BS EN 12504-4:2000. Testing Concrete. Determination of ultrasonic pulse velocity. London, 2000.

Giudice, Carlos. (2016). Corrosión Metálica y mecanismo de protección de los pigmentos inhibidores em pinturas. Notas de clases. Fortaleza: Universidade Federal do Ceará/Brasil.

Helene, P.R.L. (1976). Corrosão em armaduras para concreto armado. São Paulo: Pini.

Lourenço, M. Z.; Souza, C. A. C. (2018). Métodos de proteção e aumento da durabilidade do concreto armado. Corrosão e degradação em estruturas de concreto armado: teoria, controle e técnicas de análise e intervenção. Rio de Janeiro: Elsevier.

Pacheco, J.; Bilesky, P.; Morais, T.R.; Grando, F.; Helene, P.. (2014). Considerações sobre o Módulo de Elasticidade do Concreto. 56을 IBRACON. ISSN 2175-8182. Natal: Congresso Brasileiro do Concreto' 2014.

Pazini. E.F.; Meira, G.R. (2013). Corrosión de armaduras en estructuras de hormigón. Boletín no 6. Mérida: Alconpat Internacional.

Posetiere M.J., Gioia, C. (2017). Tecnología del Hormigón. Guia de Ensayos. Córdoba: Educo.

Ribeiro, D. V. e Cunha; M. P. T. (2018). Técnicas de avaliação e monitoramento da corrosão em estruturas de concreto armado. Corrosão e degradação em estruturas de concreto armado: teoria, controle e técnicas de análise e intervenção. Rio de Janeiro: Elsevier.

Söylev, T.A.; Richardson, M.G. (2006). Corrosion inhibitors for steel in concreto: state of the art report. Elsevier. www.sciencedirect.com.

Zerbinho R. L.; Carasco, M.F. (2012). Hormigón endurecido. Ese material llamado hormigón. Buenos Aires: Asociación Argentina de Tecnología del Hormigón. 